\title{
VARIATIONS OF CUB FILTER ON $P_{\kappa} \lambda$
}

\author{
YO MATSUBARA
}

(Communicated by Thomas J. Jech)

\begin{abstract}
In his 1973 paper, Jech extended the notion of cub and stationary sets to such sets in $P_{\kappa} \lambda$ and showed that many of their properties are preserved. We study variations of cub filters in this paper. We make use of the partition property (a large cardinal hypothesis) to investigate the properties of these filters. In the last section we investigate the relation of our filter to supercompact filters on $P_{\aleph_{1}} \lambda$ under the Axiom of Determinacy. This motivates the formulation of a certain infinitary partition property, and this property implies the $\lambda$-supercompactness of $\aleph_{1}$.
\end{abstract}

1. Closed and unbounded sets in $P_{\kappa} \lambda$. All of the notions and the results presented in this section are essentially due to Jech [4]. We begin by introducing basic notions.

DEFinition. Given a regular infinite cardinal $\kappa$ and cardinal $\lambda \geq \kappa$ we let $P_{\kappa} \lambda=\{s \subseteq \lambda:|s|<\kappa\}$, where $|s|$ is the cardinality of $s$.

Throughout this paper, we use $\kappa$ to denote a regular uncountable cardinal and $\lambda$ an ordinal $\geq \kappa$. We will be working in ZFC unless specified otherwise.

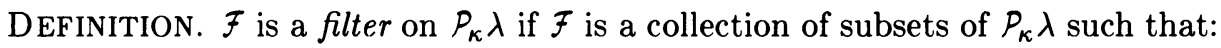

(i) $P_{\kappa} \lambda \in \mathcal{F}$ and $\varnothing \notin \mathcal{F}$.

(ii) If $X, Y \subseteq P_{\kappa} \lambda, X \in \mathcal{F}$ and $X \subseteq Y$ then $Y \in \mathcal{F}$.

(iii) If $X \in \mathcal{F}$ and $Y \in \mathcal{F}$ then $X \cap Y \in \mathcal{F}$.

A filter $\mathcal{F}$ on $P_{\kappa} \lambda$ is an ultrafilter if for any $X \subseteq P_{\kappa} \lambda, X \in \mathcal{F}$ or $P_{\kappa} \lambda-X \in \mathcal{F}$.

A filter $\mathcal{F}$ on $P_{\kappa} \lambda$ is $\delta$-complete for a cardinal $\delta \leq \kappa$ if $\mathcal{F}$ is closed under intersection of less than $\delta$ sets, i.e., if whenever $\left\{X_{\alpha}: \alpha<\beta\right\}$ is a family of members of $\mathcal{F}$ and $\beta<\delta$ then $\bigcap_{\alpha<\beta} X_{\alpha} \in \mathcal{F}$.

A filter $\mathcal{F}$ on $P_{\kappa} \lambda$ is normal if for any $\left\{X_{\alpha}: \alpha<\lambda\right\} \subseteq \mathcal{F}$ we have $\triangle_{\alpha<\lambda} X_{\alpha} \in \mathcal{F}$ where $\triangle_{\alpha<\lambda} X_{\alpha}$ is called the diagonal intersection of $\left\{X_{\alpha}: \alpha<\lambda\right\}$ and defined as follows: $s \in \triangle_{\alpha<\lambda} X_{\alpha}$ iff $s \in \bigcap_{\alpha \in s} X_{\alpha}$.

A filter $\mathcal{F}$ on $P_{\kappa} \lambda$ is fine if for each $\alpha<\lambda,\left\{s \in \mathcal{P}_{\kappa} \lambda: \alpha \in s\right\} \in \mathcal{F}$.

A filter $\mathcal{F}$ on $P_{\kappa} \lambda$ is called a supercompact filter if $\mathcal{F}$ is a $\kappa$-complete normal fine ultrafilter. A cardinal $\kappa$ is a $\lambda$-supercompact cardinal if there is a supercompact filter on $P_{\kappa} \lambda . \kappa$ is a supercompact cardinal if it is $\lambda$-supercompact for every $\lambda \geq \kappa$. Given a supercompact filter $₹$ on $P_{\kappa} \lambda$, we can define a corresponding twovalued measure $\mu$ on $P_{\kappa} \lambda$ as follows: $\mu(X)=1$ iff $X \in \mathcal{F}$. We call these measures supercompact measures on $P_{\kappa} \lambda$.

Received by the editors July 30,1986 .

1980 Mathematics Subject Classification (1985 Revision). Primary 03E05, 03E60.

The results presented in this work appeared in the author's Ph.D. thesis (UCLA, 1985). The author would like to express his gratitude to his adviser Professor D. A. Martin for his help and encouragement. 
Definition. A set $X \subseteq P_{\kappa} \lambda$ is unbounded if for any $s \in P_{\kappa} \lambda$ there exists $t \in X$ such that $s \subseteq t$. For any set $X \subseteq P_{\kappa} \lambda$ and a limit ordinal $\sigma<\kappa$ we say $\left\langle s_{\alpha}: \alpha<\sigma\right\rangle$ is a chain from $X$ if for each $\alpha<\sigma, s_{\alpha} \in X$ and $s_{\alpha} \subsetneq s_{\beta}$ if $\alpha<\beta<\sigma$.

DEFinition. Given any $X \subseteq P_{\kappa} \lambda$ we define $(X) l \cdot p \subseteq P_{\kappa} \lambda$ as follows: $s \in(X) l \cdot p$ iff $s$ is the union of some chain from $X$. A set $X \subseteq P_{\kappa} \lambda$ is closed if $(X) l \cdot p \subseteq X$. A set $X \subseteq P_{\kappa} \lambda$ is $c u b$ if $X$ is closed and unbounded. We let $\mathcal{F}_{\kappa \lambda}^{\text {cub }}=\{X \subseteq$ $P_{\kappa} \lambda$ : there exists a cub $\left.C \subseteq X\right\}$.

$\mathcal{F}_{\kappa \lambda}^{\text {cub }}$ has nice properties.

THEOREM 1 (JECH [4]). $\mathcal{F}_{\kappa \lambda}^{\text {cub }}$ is a $\kappa$-complete normal fine filter on $\mathcal{P}_{\kappa} \lambda$. $\mathcal{F}_{\kappa \lambda}^{\text {cub }}$ is called the cub filter on $\mathcal{P}_{\kappa} \lambda$.

Definition. A set $X \subseteq \mathcal{P}_{\kappa} \lambda$ is stationary if $X \cap C \neq \varnothing$ for every cub $C \subseteq \mathcal{P}_{\kappa} \lambda$.

The following theorem of Jech is an analogue of Födor's theorem.

THEOREM 2 (JECH [4]). Let $f$ be a function defined on a stationary subset of $P_{\kappa} \lambda$. If $\left\{s \in P_{\kappa} \lambda: f(s) \in s\right\}$ is stationary then $f$ is constant on some stationary set.

There is an alternate definition of cub subsets of $P_{\kappa} \lambda$. In order to present this we will introduce the following definitions.

Definition. A set $D \subseteq \mathcal{P}_{\kappa} \lambda$ is directed if for every $s, t \in D$ there is a $u \in D$ such that $s \cup t \subseteq u$.

REMARK. If $\left\langle s_{\alpha}: \alpha<\sigma\right\rangle$ is a chain then $\left\{s_{\alpha}: \alpha<\sigma\right\}$ is a directed set. Given any set $X \subseteq P_{\kappa} \lambda$ let $(X) d=\left\{s \in P_{\kappa} \lambda\right.$ : there is a directed set $D \subseteq X$ such that $|D|<$ $\kappa$ and $\bigcup D=s\}$. Solovay noticed that for any unbounded $X \subseteq P_{\kappa} \lambda, X$ is closed iff $(X) d \subseteq X$. This gives an alternate definition of cub subsets of $P_{\kappa} \lambda$ as follows: $X \subseteq P_{\kappa} \lambda$ is cub iff $X$ is unbounded and $(X) d \subseteq X$.

2. Variations of the cub filter on $P_{\kappa} \lambda$. Now we are ready to define some variations of the cub filter on $P_{\kappa} \lambda$.

DEFINITION. $\mathcal{F}_{\kappa \lambda}^{l \cdot p}=\left\{X \subseteq P_{\kappa} \lambda\right.$ : there is an unbounded $Y \subseteq P_{\kappa} \lambda$ such that $(Y) l \cdot p \subseteq X\} . \mathcal{F}_{\kappa \lambda}^{d}=\left\{X \subseteq P_{\kappa} \lambda\right.$ : there is an unbounded $Y \subseteq P_{\kappa} \lambda$ such that $(Y) d$ $\subseteq X\}$.

By the remark succeeding the definition of directness we know $(X) l \cdot p \subseteq(X) d$ for any $X \subseteq P_{\kappa} \lambda$. Thus $\mathcal{F}_{\kappa \lambda}^{d} \subseteq \mathcal{F}_{\kappa \lambda}^{l \cdot p}$. Also if $C$ is a cub subset of $P_{\kappa} \lambda$ then $C$ is unbounded and $(C) d \subseteq C$. Thus $\mathcal{F}_{\kappa \lambda}^{\text {cub }} \subseteq \mathcal{F}_{\kappa \lambda}^{d}$.

Are $\mathcal{F}_{\kappa \lambda}^{\text {cub }}, \mathcal{F}_{\kappa \lambda}^{l \cdot p}$ and $\mathcal{F}_{\kappa \lambda}^{d}$ distinct from one another? This is a natural question to ask. Before we investigate this problem, let us introduce a few more definitions.

DEFINITION. $\triangleleft \subseteq P_{\kappa} \lambda \times P_{\kappa} \lambda$ is a nice ordering if the following conditions hold:

(i) If $s \triangleleft t$ then $s \subsetneq t$.

(ii) For every $s \in \vec{P}_{\kappa} \lambda$ there is $t \in P_{\kappa} \lambda$ such that $s \triangleleft t$.

(iii) For every $s, t$ and $u$ if $s \triangleleft t$ and $t \subseteq u$ then $s \triangleleft u$.

(iv) For every $s, t$ and $u$ if $s \subseteq t$ and $t \triangleleft u$ then $s \triangleleft u$.

REMARK. $\subsetneq$ is a nice ordering. From now on we always assume $\triangleleft$ is a nice ordering. We say $\left\langle s_{\alpha}: \alpha<\sigma\right\rangle$ is a $\triangleleft$-chain if $s_{\alpha} \triangleleft s_{\beta}$ for every $\alpha<\beta<\sigma$.

DEFINITION. For $X \subseteq P_{\kappa} \lambda$ we let $(X) \triangleleft l \cdot p=\left\{s \in P_{\kappa} \lambda: s\right.$ is the union of some $\triangleleft$-chain from $X$ \}.

$X \subseteq P_{\kappa} \lambda$ is $\triangleleft-c u b$ iff $X$ is unbounded and $(X) \triangleleft l \cdot p \subseteq X$. 
$\mathcal{F}_{\kappa \lambda}^{\triangleleft \mathrm{cub}}=\left\{X \subseteq P_{\kappa} \lambda:\right.$ there is a $\triangleleft$-cub $\left.C \subseteq X\right\}$.

$\mathcal{F}_{\kappa \lambda}^{\triangleleft l \cdot p}=\left\{X \subseteq P_{\kappa} \lambda\right.$ : there is an unbounded $Y \subseteq P_{\kappa} \lambda$ such that $\left.(Y) \triangleleft l \cdot p \subseteq X\right\}$.

We say $X \subseteq P_{\kappa} \lambda$ is $\triangleleft$-directed if for every $s, t \in X$ there is some $u \in X$ such that $s \cup t \triangleleft u$. For $X \subseteq P_{\kappa} \lambda$ we let $(X) \triangleleft d=\left\{s \in P_{\kappa} \lambda\right.$ : there is a $\triangleleft$-directed set $D \subseteq X$ such that $|D|<\kappa$ and $\bigcup D=s\}$.

$\mathcal{F}_{\kappa \lambda}^{\triangleleft d}=\left\{X \subseteq P_{\kappa} \lambda\right.$ : there is an unbounded $Y \subseteq P_{\kappa} \lambda$ such that $\left.(Y) \triangleleft d \subseteq X\right\}$.

As before we see $\mathcal{F}_{\kappa \lambda}^{\triangleleft c u b} \subseteq \mathcal{F}_{\kappa \lambda}^{\triangleleft d} \subseteq \mathcal{F}_{\kappa \lambda}^{\triangleleft l \cdot p}$. Furthermore it is clear that $\mathcal{F}_{\kappa \lambda}^{\text {cub }} \subseteq$ $\mathcal{F}_{\kappa \lambda}^{\triangleleft c u b}, \mathcal{F}_{\kappa \lambda}^{d} \subseteq \mathcal{F}_{\kappa \lambda}^{\triangleleft d}$ and $\mathcal{F}_{\kappa \lambda}^{l \cdot p} \subseteq \mathcal{F}_{\kappa \lambda}^{\triangleleft l \cdot p}$. Now we study the relationship among the $\mathcal{F}_{\kappa \lambda}$ 's. First for $\kappa=\aleph_{1}$ we have the following:

LEMMA 3. (i) $\mathcal{F}_{\aleph_{1} \lambda}^{d}=\mathcal{F}_{\aleph_{1} \lambda}^{l \cdot p}$.

(ii) For every nice $\triangleleft$ we have $\mathcal{F}_{\aleph_{1} \lambda}^{\triangleleft d}=\mathcal{F}_{\aleph_{1} \lambda}^{\triangleleft l \cdot p}$.

PROOF. It is easy to see that $(X) l \cdot p=(X) d$ for $X \subseteq P_{\aleph_{1}} \lambda$. Thus by the definition of $\digamma_{N_{1} \lambda}$ 's, we have (i) and (ii).

Even if $\kappa>\aleph_{1}$, we still have the following.

THEOREM 4. $\mathcal{F}_{\kappa \lambda}^{\text {cub }}=\mathcal{F}_{\kappa \lambda}^{d}=\mathcal{F}_{\kappa \lambda}^{\triangleleft d}$ for every regular cardinal $\kappa \leq \lambda$ and nice ordering $\triangleleft$.

PROOF. Since we already know $\mathcal{F}_{\kappa \lambda}^{\text {cub }} \subseteq \mathcal{F}_{\kappa \lambda}^{d} \subseteq \mathcal{F}_{\kappa \lambda}^{\triangleleft d}$ it suffices to show that $\mathcal{F}_{\kappa \lambda}^{\triangleleft d} \subseteq \mathcal{F}_{\kappa \lambda}^{c u b}$.

Suppose we have some function $f:[\lambda]^{<\omega} \rightarrow \mathcal{P}_{\kappa} \lambda$; we let $A_{f}=\left\{s \in \mathcal{P}_{\kappa} \lambda\right.$ : for any $\left.a \in[s]^{<\omega}, f(a) \subseteq s\right\}$. It is easy to see that $A_{f}$ is cub for any $f:[\lambda]^{<\omega} \rightarrow P_{\kappa} \lambda .{ }^{1}$ For any unbounded $X \subseteq \rho_{\kappa} \lambda$, we will find some $f:[\lambda]^{<\omega} \rightarrow \rho_{\kappa} \lambda$ such that $A_{f} \subseteq(X) \triangleleft d$. This will show $\mathcal{F}_{\kappa \lambda}^{\triangleleft d} \subseteq \mathcal{F}_{\kappa \lambda}^{\text {cub }}$.

Now fix $\triangleleft$ a nice ordering and $X$ an unbounded subset of $P_{\kappa} \lambda$. We will define the value of $f$ at $\left\{\alpha_{0}, \ldots, \alpha_{n}\right\}$ where $\alpha_{0}<\cdots<\alpha_{n}<\lambda$ by induction on $n$. Let $f\left(\left\{\alpha_{0}\right\}\right)$ be any infinite $s \in X$ such that $\alpha_{0} \in s$. Given $f\left(\left\{\beta_{0} \cdots \beta_{n}\right\}\right)$ for every $\beta_{0}<\cdots<\beta_{n}<\lambda$, let $f\left(\left\{\alpha_{0} \cdots \alpha_{n+1}\right\}\right)$ be any $t \in X$ such that

$$
\bigcup\left\{f(a): a \subseteq\left\{\alpha_{0} \cdots \alpha_{n+1}\right\} \text { and }|a| \leq n+1\right\} \triangleleft t .
$$

Claim. $A_{f} \subseteq(X) \triangleleft d$.

ProOF. Let $s \in A_{f}$. So $\bigcup\left\{f(a): a \in[s]^{<\omega}\right\} \subseteq s$. But since $\alpha \in f(\{\alpha\})$ for each $\alpha<\lambda$ we have $\bigcup\left\{f(a): a \in[s]^{<\omega}\right\}=s$. It is easy to see that $\left\{f(a): a \in[s]^{<\omega}\right\}$ is a $\triangleleft$-directed subset of $X$. Thus $s \in(X) \triangleleft d$.

By combining Lemma 3 and Theorem 4 we obtain

COROLLARY 5. $\mathcal{F}_{\aleph_{1} \lambda}^{\text {cub }}=\mathcal{F}_{\aleph_{1} \lambda}^{d}=\mathcal{F}_{\aleph_{1} \lambda}^{l \cdot p}=\mathcal{F}_{\aleph_{1} \lambda}^{\triangleleft \text { cub }}=\mathcal{F}_{\aleph_{1} \lambda}^{\triangleleft d}=\mathcal{F}_{\aleph_{1} \lambda}^{\triangleleft l \cdot p}$ for any nice ordering $\triangleleft$ on $P_{\aleph_{1}} \lambda$.

From the above results, one might expect to prove that all these $\mathcal{F}_{\kappa \lambda}$ 's are identical for every regular $\kappa$. But this turns out not to be the case.

THEOREM 6 (ZWICKER [13]). If $\kappa$ is a supercompact cardinal then $\mathcal{F}_{\kappa \lambda}^{\text {cub }} \neq$ $\mathcal{F}_{\kappa \lambda}^{l \cdot p}$ for any cardinal $\lambda>\kappa$.

PROOF. We need the following lemma whose proof will be given in the next section.

${ }^{1}$ This was used by Kueker in [6] and by Menas in [9]. 
LEMMA 7. If $\kappa$ is a supercompact cardinal then $\left\{s \in \mathcal{P}_{\kappa} \lambda:|s \cap \kappa|=|s|\right\} \in \mathcal{F}_{\kappa \lambda}^{l \cdot p}$ for any cardinal $\lambda>\kappa$.

Let $\mathcal{F}$ be a supercompact filter. It is well known that $\mathcal{F}$ extends $\mathcal{F}_{\kappa \lambda}^{\text {cub }}$. In $V^{P_{\kappa} \lambda} / \mathcal{F}$, $s \mapsto|s \cap \kappa|$ represents $\kappa$ and $s \mapsto|s|$ represents $\lambda$. Thus $\left\{s \in \mathcal{P}_{\kappa} \lambda:|s \cap \kappa|<|s|\right\} \in \mathcal{F}$. This gives $\mathcal{F}_{\kappa \lambda}^{\text {cub }} \neq \mathcal{F}_{\kappa \lambda}^{l \cdot p}$. $\quad$ Mod Lemma 7

3. Partition properties on $P_{\kappa} \lambda$. By Theorem 1 we know $\mathcal{F}_{\kappa \lambda}^{\text {cub }}$ is a $\kappa$-complete normal filter. We would like to ask the same question about $\mathcal{F}_{\kappa \lambda}^{l \cdot p}$. It appears to be that unless some conditions on $\kappa$ and $\lambda$ are imposed, there is no guarantee that $\mathcal{F}_{\kappa \lambda}^{l \cdot p}$ forms a filter let alone that it is $\kappa$-complete and normal. Nevertheless there is a large cardinal hypothesis which guarantees the $\kappa$-completeness and the normality of $\mathcal{F}_{\kappa \lambda}^{l \cdot p}$. Before we introduce this hypothesis, we need a few preliminary notations.

Notations. Given any $X \subseteq P_{\kappa} \lambda$ and nice ordering $\triangleleft$ on $P_{\kappa} \lambda$, let $[X]_{\triangleleft}^{2}=$ $\{\langle s, t\rangle: s, t \in X$ and $s \triangleleft t\}$ and $[X]^{2}=[X]_{\subsetneq}^{2}$.

DeFinition (PARTITION PROPERTIES ON $P_{\kappa} \lambda$ ). By $P_{\kappa} \lambda \rightarrow\left(\right.$ Unbounded) ${ }_{2}^{\triangleleft 2}$ we mean that given any $F:\left[P_{\kappa} \lambda\right]^{2} \rightarrow 2$ there is an unbounded $X \subseteq P_{\kappa} \lambda$, called a homogeneous set, such that $\left|F^{\prime \prime}[X]_{\triangleleft}^{2}\right|=1$. By $P_{\kappa} \lambda \rightarrow$ (Unbounded) ${ }_{2}^{2}$ we mean $P_{\kappa} \lambda \rightarrow$ (Unbounded) $)_{2}^{\subsetneq 2}$. By $P_{\kappa} \lambda \rightarrow\left(\right.$ Stationary) ${ }_{2}^{\triangleleft 2}$ we mean given any $F:\left[P_{\kappa} \lambda\right]^{\triangleleft 2} \rightarrow 2$ there is a stationary homogeneous set for $F$, and $P_{\kappa} \lambda \rightarrow(\text { Stationary })_{2}^{\Upsilon_{2}^{2}}$ is denoted by $P_{\kappa} \lambda \rightarrow$ (Stationary $)_{2}^{2}$.

REMARK. It is easy to see that

$$
P_{\kappa} \lambda \rightarrow(\text { Unbounded })_{2}^{2} \text { implies } \quad P_{\kappa} \lambda \rightarrow(\text { Unbounded })_{2}^{\triangleleft 2}
$$

for every nice ordering $\triangleleft$.

These partition properties were introduced by Jech [4]. He observed that they are large cardinal hypotheses.

THEOREM 8 ( JECH [4]). If $P_{\kappa} \lambda \rightarrow(\text { Unbounded })_{2}^{2}$ then $\kappa$ is a weakly compact cardinal.

Actually his proof shows that if $P_{\kappa} \lambda \rightarrow$ (Unbounded) ${ }_{2}^{\triangleleft 2}$ for a nice ordering $\triangleleft$ then $\kappa$ is a weakly compact cardinal. Magidor [7] has an analogous result for $P_{\kappa} \lambda \rightarrow$ (Stationary $)_{2}^{2}$.

Thus the partition properties on $P_{\kappa} \lambda$ imply the large cardinal hypotheses. Is there any large cardinal hypothesis which will imply the partition property? Menas has the following:

THEOREM 9 (MENAS [10]). If $\kappa$ is a supercompact cardinal then $\mathcal{P}_{\kappa} \lambda \rightarrow$ (Stationary) ${ }_{2}^{2}$ holds for every $\lambda \geq \kappa$.

Menas actually proved: if $\kappa$ is a supercompact cardinal then for each $\lambda \geq \kappa$ there is a $\lambda$-supercompact filter $\mathcal{F}$ on $P_{\kappa} \lambda$ such that every $F:\left[P_{\kappa} \lambda\right]^{2} \rightarrow 2$ will have a homogeneous set in $\mathcal{F}$. This gives the above theorem since every set in $\mathcal{F}$ is stationary.

Let us now apply the partition properties to study the nature of $\mathcal{F}_{\kappa \lambda}^{l \cdot p}$.

THEOREM 10. (i) If $P_{\kappa} \lambda \rightarrow$ (Unbounded) ${ }_{2}^{2}$ then $\mathcal{F}_{\kappa \lambda}^{l \cdot p}$ is a $\kappa$-complete normal fine filter on $P_{\kappa} \lambda$. 
(ii) If $P_{\kappa} \lambda \rightarrow(\text { Unbounded })_{2}^{\triangleleft 2}$ for some nice ordering $\triangleleft$ on $P_{\kappa} \lambda$ then $\mathcal{F}_{\kappa \lambda}^{\triangleleft l \cdot p}$ is a $\kappa$-complete normal fine filter on $P_{\kappa} \lambda$.

PrOOF. We will only prove (i). The proof of (ii) is identical.

Assume $P_{\kappa} \lambda \rightarrow$ (Unbounded) ${ }_{2}^{2}$. We will first show $\mathcal{F}_{\kappa \lambda}^{l \cdot p}$ is $\kappa$-complete. Suppose $\left\{X_{\alpha}: \alpha<\gamma\right\} \subseteq \mathcal{F}_{\kappa \lambda}^{l \cdot p}$ where $\gamma<\kappa$. By the definition of $\mathcal{F}_{\kappa \lambda}^{l \cdot p}$, for each $\alpha<\gamma$ there is an unbounded $Y_{\alpha} \subseteq P_{\kappa} \lambda$ such that $\left(Y_{\alpha}\right) l \cdot p \subseteq X_{\alpha}$. Define $F:\left[P_{\kappa} \lambda\right]^{2} \rightarrow 2$ as follows:

$$
F(s, t)= \begin{cases}0 & \text { if for each } \alpha<\gamma \text { there is some } u_{\alpha} \in Y_{\alpha} \\ & \text { such that } s \subsetneq u_{\alpha} \subsetneq t, \\ 1 & \text { otherwise. }\end{cases}
$$

By the partition property there is an unbounded homogeneous set, say $H$, for our $F$. It is easy to see that $F^{\prime \prime}[H]^{2}=\{0\}$. This guarantee $(H) l \cdot p \subseteq \bigcap_{\alpha<\gamma}\left(Y_{\alpha}\right) l \cdot p$. Thus $\bigcap_{\alpha<\gamma} X_{\alpha} \in \mathcal{F}_{\kappa \lambda}^{l \cdot p}$.

Normality. Suppose $\left\{X_{\alpha}: \alpha<\lambda\right\} \subseteq P_{\kappa} \lambda$. We want to show that $\triangle_{\alpha<\gamma} X_{\alpha} \in$ $F_{\kappa \lambda}^{l \cdot p}$. For each $\alpha<\gamma$ we have an unbounded set $Y_{\alpha} \subseteq P_{\kappa} \lambda$ such that $\left(Y_{\alpha}\right) l \cdot p \subseteq X_{\alpha}$. Define $G:\left[P_{\kappa} \lambda\right]^{2} \rightarrow 2$ as follows:

$$
G(s, t)= \begin{cases}0 & \text { if for every } \alpha \in s \text { there exists } u_{\alpha} \in Y_{\alpha} \\ \text { such that } s \subsetneq u_{\alpha} \subsetneq t & \text { otherwise. }\end{cases}
$$

Let $H$ be an unbounded homogeneous set for $G$. It is easy to see $G^{\prime \prime}[H]^{2}=\{0\}$. By the way $G$ was defined we have $(H) l \cdot p \subseteq \triangle_{\alpha<\gamma} X_{\alpha}$. Thus $\triangle_{\alpha<\gamma} X_{\alpha} \in \mathcal{F}_{\kappa \lambda}^{l \cdot p}$. The fineness is trivial.

So, under the partition property, $\mathcal{F}_{\kappa \lambda}^{l \cdot p}$ is quite well behaved as a filter. Actually it turns out that, under $P_{\kappa} \lambda \rightarrow(\text { Unbounded })_{2}^{2}, \mathcal{F}_{\kappa \lambda}^{l \cdot p}$ and $\mathcal{F}_{\kappa \lambda}^{\triangleleft l \cdot p}$ for any nice ordering $\triangleleft$ are the same. $\triangleleft$.

THEOREM 11. If $\mathcal{P}_{\kappa} \lambda \rightarrow(\text { Unbounded })_{2}^{2}$ then $\mathcal{F}_{\kappa \lambda}^{\triangleleft l \cdot p}=\mathcal{F}_{\kappa \lambda}^{l \cdot p}$ for any nice ordering

PROOF. We already know $\mathcal{F}_{\kappa \lambda}^{l \cdot p} \subseteq \mathcal{F}_{\kappa \lambda}^{\triangleleft l \cdot p}$ for any nice $\triangleleft$. Let $X \in \mathcal{F}_{\kappa \lambda}^{\triangleleft l \cdot p}$. There is an unbounded $Y \subseteq P_{\kappa} \lambda$ such that $(Y) \triangleleft l \cdot p \subseteq X$. Define $F:\left[P_{\kappa} \lambda\right]^{2} \rightarrow 2$ as follows:

$$
F(s, t)= \begin{cases}0 & \text { if there is some } u \in Y \text { such that } s \triangleleft u \triangleleft t, \\ 1 & \text { otherwise. }\end{cases}
$$

Let $H$ be an unbounded homogeneous set for $F$. It is easy to see that $F^{\prime \prime}[H]^{2}=\{0\}$. By the way $F$ was defined we have $(H) l \cdot p \subseteq(Y) \triangleleft l \cdot p$. So $X \in \mathcal{F}_{\kappa \lambda}^{l \cdot p}$. Thus $\mathcal{F}_{\kappa \lambda}^{\triangleleft l \cdot p}=\mathcal{F}_{\kappa \lambda}^{l \cdot p}$.

We have postponed the proof of Lemma 7. The following lemma together with Theorem 9 proves Lemma 7.

THEOREM 12. If $P_{\kappa} \lambda \rightarrow$ (Unbounded) $)_{2}^{2}$ then $\left\{s \in P_{\kappa} \lambda:|s \cap \kappa|=|s|\right\} \in \mathcal{F}_{\kappa \lambda}^{l \cdot p}$.

ProOF. Define $F:\left[P_{\kappa} \lambda\right]^{2} \rightarrow 2$ as follows:

$$
F(s, t)= \begin{cases}0 & \text { if }|s|<|t \cap \kappa| \\ 1 & \text { otherwise }\end{cases}
$$


Let $H$ be an unbounded homogeneous set for $F$. Clearly $F^{\prime \prime}[H]^{2}=\{0\}$. It is easy to see that $(H) l \cdot p \subseteq\left\{s \in P_{\kappa} \lambda:|s \cap \kappa|=|s|\right\}$. Thus $\left\{s \in \mathcal{P}_{\kappa} \lambda:|s \cap \kappa|=|s|\right\} \in \mathcal{F}_{\kappa \lambda}^{l \cdot p}$.

Under the partition property if we further assume that $\lambda$ is a Ramsey cardinal then we can carry out Zwicker's argument to prove $\mathcal{F}_{\kappa \lambda}^{\text {cub }} \neq \mathcal{F}_{\kappa \lambda}^{l \cdot p}$. The key step is the following result of Baldwin.

LEMMA 13 (BALDWIN [1]). If $\lambda$ is Ramsey and $\kappa$ strongly inaccessible $<\lambda$, then $\left\{s \in P_{\kappa} \lambda:|s \cap \kappa|<|s|\right\}$ is stationary.

REMARK. Baldwin uses a slightly weaker hypothesis than $\lambda$ Ramsey.

THEOREM 14 (VIA BALDWIN). If $\lambda$ is a Ramsey cardinal $>\kappa$ and $P_{\kappa} \lambda$ $\rightarrow(\text { Unbounded })_{2}^{2}$, then $\mathcal{F}_{\kappa \lambda}^{\text {cub }} \neq \mathcal{F}_{\kappa \lambda}^{l \cdot p}$.

ProOF. By Lemma 12 we know $\left\{s \in \mathcal{P}_{\kappa} \lambda:|s \cap \kappa|=|s|\right\} \in \mathcal{F}_{\kappa \lambda}^{l \cdot p}$. But by Lemma 13 we have $\left\{s \in \mathcal{P}_{\kappa} \lambda:|s \cap \kappa|<|s|\right\}$ is stationary. Thus $\mathcal{F}_{\kappa \lambda}^{l \cdot p} \neq \mathcal{F}_{\kappa \lambda}^{\text {cub }}$.

$\mathcal{F}_{\kappa \lambda}^{l \cdot p}=\mathcal{F}_{\kappa \lambda}^{\text {cub }}$ has an interesting consequence.

THEOREM 15. If $\mathcal{F}_{\kappa \lambda}^{l \cdot p}=\mathcal{F}_{\kappa \lambda}^{\text {cub }}$, then every stationary subset of $\mathcal{P}_{\kappa} \lambda$ can be decomposed into $\lambda$ many disjoint stationary subsets.

ProOF. See [8].

4. AD world: The supercompactness of $\aleph_{1}$. So far we have been working in the classical axioms of set theory known as ZFC (Zermelo-Frankel Axioms with the Axiom of Choice). Since the late 1960's, many set theorists have been investigating the consequences of the Axiom of Determinacy (denoted by $\mathrm{AD}$ ). One of the interesting features of set theory with $\mathrm{AD}$ is that we can prove the existence of cardinals satisfying properties which have been known as "large cardinal axioms". Furthermore in this theory, those cardinals satisfying the large cardinal axioms are not necessarily large.

For example, Solovay showed that $\aleph_{1}$ is a measurable cardinal under AD. Along this line Harrington, Kechris and Solovay have the following result:

THEOREM 16 (HARRINGTON, KECHRIS AND SOLOVAY (HARRINGTON, KECHRIS [3])). Assume AD and DC (the Axiom of Dependent Choice). If $\lambda$ lies below some Suslin cardinal, then $\aleph_{1}$ is a $\lambda$-supercompact cardinal.

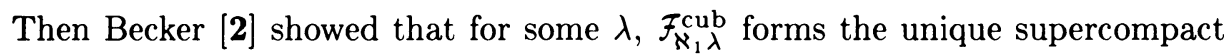
filter on $P_{\aleph_{1}} \lambda$.

THEOREM 17 (BECKER). ZF + AD + DC. Let $\lambda$ be a semireliable projective ordinal. Then $\mathcal{F}_{\aleph_{1} \lambda}^{\text {cub }}$ is the unique supercompact filter on $P_{\aleph_{1}} \lambda$.

Recently Woodin [12] proved the uniqueness of supercompact filter on $P_{\aleph_{1}} \lambda$ for many $\lambda$.

THEOREM 18 (WOODIN). ZF $+\mathrm{AD}+\mathrm{DC}$. If $\lambda \leq$ some Suslin cardinal, then the supercompact filter on $P_{\aleph_{1}} \lambda$ is unique.

Assuming $\lambda$-supercompactness of $\aleph_{1}$, we are interested in the relationship between $\mathcal{F}_{\mathcal{N}_{1} \lambda}^{l \cdot p}$ and the supercompact filters on $\mathcal{P}_{\aleph_{1}} \lambda$. We have the following result: 
THEOREM 19. ZF + DC. For any ordinal $\lambda \geq \aleph_{1}$, if $\mathcal{F}$ is a $\lambda$-supercompact filter on $\mathcal{P}_{\aleph_{1}} \lambda$ then $\mathcal{F}$ extends $\mathcal{F}_{\aleph_{1} \lambda}^{l \cdot p}$.

PROOF. The proof is modeled after the proof of Theorem 18. Let $\mu$ be a supercompact measure on $P_{\aleph_{1}} \lambda$. Let $X \subseteq P_{\aleph_{1}} \lambda$ be an unbounded set. It suffices to show that $\mu((X) l \cdot p)=1$.

For each $s \in P_{\aleph_{1}} \lambda$, we will define the game $G_{s}$ as follows:

$$
\begin{array}{cccccc}
\text { I } & \alpha_{0} & & \alpha_{1} & & \cdots \\
\text { II } & & t_{0} & & t_{1} & \cdots
\end{array}
$$

Player I plays ordinals $\alpha_{i} \in s$. Player II plays $t_{i} \in X$, a subset of $s$, such that $\alpha_{i} \in t_{i}$ and $t_{i-1} \subsetneq t_{i}$. Player I wins iff at some state Player II cannot play anymore.

Clearly $G_{s}$ is an open game for Player I. Thus either I or II has a winning "quasi-strategy". See [11] for the definition of quasi-strategy.

ClaIM. $\mu\left\{s \in \mathcal{P}_{\aleph_{1}} \lambda\right.$ : II wins the game $\left.G_{s}\right\}=1$.

ProOF. Suppose not, i.e., $\mu\left\{s \in \mathcal{P}_{\aleph_{1}} \lambda\right.$ : I wins $\left.G_{s}\right\}=1$. But if I wins $G_{s}$, it has the canonical winning strategy, call this $\tau_{s}$. View $\tau_{s}$ as a set of (legal) position in $G_{s}$.

Suppose $q=\left\langle\alpha_{0}, t_{0}, \ldots, \alpha_{i}, t_{i}\right\rangle$ is a sequence such that $q \in \tau_{s}$ for $\mu$-almost all $s$. Hence for each such $s$, there is an $\alpha \in s$ such that $q^{\cap}\langle\alpha\rangle \in \tau_{s}$. This defines a function $f: P_{\aleph_{1}} \lambda \rightarrow \lambda$ with $f(s) \in s$ on a set of $\mu$-measure one. So there is an $\alpha_{i+1}<\lambda$ such that $q^{\cap}\left\langle\alpha_{i+1}\right\rangle \in \tau_{s}$ for $\mu$-almost all $s$. Pick $t_{i+1} \in X$ such that $\alpha_{i+1} \in t_{i+1}$ and $t_{i} \subsetneq t_{i+1}$. Let $A=\left\{s \in \mathcal{P}_{\aleph_{1}} \lambda: q^{\cap}\left\langle\alpha_{i+1}\right\rangle \in \tau_{s}\right.$ and $\left.s \supseteq t_{i+1}\right\}$. Clearly $\mu(A)=1$, and for any $s \in A, q^{\cap}\left\langle\alpha_{i+1}, t_{i+1}\right\rangle \in \tau_{s}$. Thus we have $\mu\{s \in$ $\left.P_{\aleph_{1}} \lambda: q^{\cap}\left\langle\alpha_{i+1}, t_{i+1}\right\rangle \in \tau_{s}\right\}=1$.

Repeating the above procedure we obtain a sequence $\left\langle\alpha_{0} t_{0} \alpha_{1} t_{1} \cdots \alpha_{i} t_{i} \cdots\right\rangle$ such that $\mu\left\{s \in \mathcal{P}_{\aleph_{1}} \lambda:\left\langle\alpha_{0} t_{0} \cdots \alpha_{i} t_{i}\right\rangle \in \tau_{s}\right\}=1$ for all $i$. So by the countable completeness of $\mu$, there is some fixed $\tau_{s^{*}}$ such that $\left\langle\alpha_{0} t_{0} \cdots \alpha_{i} t_{i}\right\rangle \in \tau_{s^{*}}$ for all $i$. Thus Player I does not win the game. Contradiction. Q.E.D. Claim

It now suffices to show that $\left\{s \in \mathcal{P}_{\aleph_{1}} \lambda\right.$ : II wins $\left.G_{s}\right\} \subseteq(X) l \cdot p$. Suppose II wins $G_{s}$. Let I play an enumeration of $s$. II's moves guarantee that $s \in(X) 1 \cdot p$.

COROllaRY 20. ZF + DC. For any ordinal $\lambda \geq \aleph_{1}$, if $\mu$ is a supercompact measure on $\mathcal{P}_{\aleph_{1}} \lambda$ then $\mu(C)=1$ for any cub $C \subseteq \mathcal{P}_{\aleph_{1}} \lambda$.

Now we will introduce a certain kind of infinitary partition property on $P_{\aleph_{1}} \lambda$.

Notation. Given any $Y \subseteq P_{\kappa} \lambda$, let $[Y]^{\omega}=\left\{\left\langle s_{n}: n \in \omega\right\rangle:\left\langle s_{n}: n \in \omega\right\rangle\right.$ is a chain from $Y$ \}.

DEFINITION. $P_{\aleph_{1}} \lambda \rightarrow$ (Unbounded) $)_{2}^{\omega}$ means given any $F:\left[P_{\aleph_{1}} \lambda\right]^{\omega} \rightarrow 2$ there is an unbounded set $X \subseteq P_{\aleph_{1}} \lambda$ such that $\left|F^{\prime \prime}[X]^{\omega}\right|=1$.

THEOREM 21. ZF + DC. If $P_{\aleph_{1}} \lambda \rightarrow$ (Unbounded) $)_{2}^{\omega}$ holds, then $\aleph_{1}$ is a $\lambda$ supercompact cardinal with a unique $\lambda$-supercompact filter.

PROOF. Assume $P_{\aleph_{1}} \lambda \rightarrow$ (Unbounded) ${ }_{2}^{\omega}$. We will show that $\mathcal{F}_{\aleph_{1} \lambda}^{l \cdot p}$ is a supercompact filter on $\mathcal{P}_{\aleph_{1}} \lambda$.

First we will show that $\mathcal{F}_{\aleph_{1} \lambda}^{l \cdot p}$ is an ultrafilter on $P_{\aleph_{1}} \lambda$. Given $X \subseteq P_{\aleph_{1}} \lambda$, define $F:\left[P_{\aleph_{1}} \lambda\right]^{\omega} \rightarrow 2$ as follows:

$$
F\left(\left\langle s_{n}: n \in \omega\right\rangle\right)= \begin{cases}0 & \text { if } \bigcup_{n} s_{n} \in X \\ 1 & \text { otherwise }\end{cases}
$$


Let $H$ be an unbounded homogeneous set for this $F$. If $F^{\prime \prime}[H]^{\omega}=\{0\}$ then it is easy to see that $(H) l \cdot p \subseteq X$. Also if $F^{\prime \prime}[H]^{\omega}=\{1\}$, then $(H) l \cdot p \subseteq \mathcal{P}_{\aleph_{1}} \lambda-X$. Thus $\mathcal{F}_{\aleph_{1} \lambda}^{l \cdot p}$ is an ultrafilter.

Now we prove that $\mathcal{F}_{\mathcal{N}_{1} \lambda}^{l \cdot p}$ is countably complete. We can carry out a proof identical to the one of $\kappa$-completeness of $\mathcal{F}_{\kappa \lambda}^{l \cdot p}$ from $P_{\kappa} \lambda \rightarrow$ (Unbounded) ${ }_{2}^{2}$ in Theorem 10. To do this we only need the countable choice.

The fineness is trivial. We now prove the normality of $\mathcal{F}_{\mathcal{N}_{1} \lambda}^{1 \cdot p}$. Assume $\{s \in$ $\left.\mathcal{P}_{\aleph_{1}} \lambda: f(s) \in s\right\} \in \mathcal{F}_{\aleph_{1} \lambda}^{l \cdot p}$. Let $X \subseteq \mathcal{P}_{\aleph_{1}} \lambda$ be an unbounded set witnessing this fact, i.e., $(X) l \cdot p \subseteq\left\{s \in \mathcal{P}_{\aleph_{1}} \lambda: f(s) \in s\right\}$. Define $F:\left[\mathcal{P}_{\aleph_{1}} \lambda\right]^{\omega} \rightarrow 2$ as follows:

$$
F\left(\left\langle s_{n}: n \in \omega\right\rangle\right)= \begin{cases}0 & \text { if } f\left(\bigcup_{n} s_{n}\right) \in s_{0} \\ 1 & \text { otherwise. }\end{cases}
$$

Let $H \subseteq P_{\aleph_{1}} \lambda$ be an unbounded homogeneous set for $F$.

ClaIM. $F^{\prime \prime}[H]^{\omega}=\{0\}$.

By DC we can pick $\left\langle s_{n}: n \in \omega\right\rangle \in[H]^{\omega}$ such that for each $n$ there exists $u_{n} \in X$ with $s_{n} \subsetneq u_{n} \subsetneq s_{n+1}$. Thus $\bigcup_{n} s_{n} \in(X) l \cdot p$. So $f\left(\bigcup_{n} s_{n}\right) \in \bigcup_{n} s_{n}$. Let $m$ be the least integer $m$ such that $f\left(\bigcup_{n} s_{n}\right) \in s_{m}$. Let $t_{0}=s_{m}, t_{1}=s_{m+1}, \ldots, t_{n}=$ $s_{m+n}, \ldots$ Then clearly $\left\langle t_{n}: n \in \omega\right\rangle \in[H]^{\omega}$ and $f\left(\bigcup_{n} t_{n}\right) \in t_{0}$. Q.E.D. Claim

Pick $s^{*} \in H$. Let $H^{*}=\left\{s \in H: s \supsetneq s^{*}\right\}$. Thus $H^{*}$ is also unbounded. By the above claim $\left(H^{*}\right) l \cdot p \subseteq\left\{s \in \mathcal{P}_{\aleph_{1}} \lambda: f(x) \in s^{*}\right\}$. But by the countable completeness of $\mathcal{F}_{\mathcal{N}_{1} \lambda}^{l \cdot p}$, we have $\left\{s \in \mathcal{P}_{\aleph_{1}} \lambda: f(s)=\alpha\right\} \in \mathcal{F}_{\aleph_{1} \lambda}^{l \cdot p}$ for some $\alpha \in s^{*}$.

The uniqueness of the supercompact filter is given by Theorem 19 .

Theorem 21 was inspired by the following theorem of Kleinberg [5]: If $\kappa>\delta$ are regular cardinals and $\kappa \rightarrow(\kappa)^{\delta+\delta}$, then $\kappa$ is a measurable cardinal. Martin proved that $\aleph_{1} \rightarrow\left(\aleph_{1}\right)^{\aleph_{1}}$ follows from $\mathrm{ZF}+\mathrm{AD}+\mathrm{DC}$. Can one prove ${\mathcal{\aleph}_{1}}_{\aleph_{1}} \rightarrow$ (Unbounded) $)_{2}^{\omega}$ from $\mathrm{ZF}+\mathrm{AD}+\mathrm{DC}$ for some $\lambda \geq \aleph_{1}$ ? It is not difficult to see that $\mathrm{ZF}+\mathrm{AD}+\mathrm{DC} \vdash P_{\aleph_{1}} \lambda \rightarrow$ (Unbounded) ${ }_{2}^{\omega}$ for $\lambda<\aleph_{2}$. But nothing is known about $P_{\aleph_{1}} \lambda \rightarrow$ (Unbounded) ${ }_{2}^{\omega}$ for $\lambda \geq \aleph_{2}$.

\section{REFERENCES}

1. Stewart Baldwin, The consistency strength of certain stationary subsets of $P_{\kappa} \lambda$, Proc. Amer. Math. Soc. 92 (1984), 90-92.

2. Howard Becker, AD and the supercompactness of $\aleph_{1}$, J. Symbolic Logic 46 (1981), 822-842.

3. Leo A. Harrington and Alexander S. Kechris, On the determinacy of games on ordinals, Ann. Math. Logic 20 (1980), 109-154.

4. Thomas J. Jech, Some combinatorial problems concerning uncountable cardinals, Ann. Math. Logic 5 (1973), 165-198.

5. Eugene M. Kleinberg, Strong partition properties for infinite cardinals, J. Symbolic Logic $\mathbf{3 5}$ (1970), 410-428.

6. David W. Kueker, Countable approximations and Löwenheim-Skolem theories, Ann. Math. Logic 11 (1977), 57-103.

7. Menachem K. Magidor, Combinatorial characterization of supercompact cardinals, Proc. Amer. Math. Soc. 42 (1974), 279-285.

8. Yo Matsubara, Menas' conjecture and generic ultrapowers, Ann. Pure Appl. Logic 36 (1987), 225-234.

9. Telis K. Menas, On strong compactness and supercompactness, Ann. Math. Logic 7 (1974), 327-359.

10.

11. Yiannis N. Moschovakis, Descriptive set theory, North-Holland, 1980. 
12. W. Hugh Woodin, $\mathrm{AD}$ and the uniqueness of the supercompact measures on $P_{\omega_{1}} \lambda$, Cabal Seminar 79-81, Lecture Notes in Math., vol. 1019, Springer-Verlag, 1983.

13. W. S. Zwicker, $P_{\kappa} \lambda$ combinatorics II: the RK ordering beneath a supercompact measure, J. Symbolic Logic 51 (1986), 604-616.

Department of Mathematics, Amherst College, Amherst, Massachusetts 01002

Current address: Department of Mathematics, Lehigh University, Bethlehem, Pennsylvania 18015 